\title{
Automated Analysis of Muscle X-ray Diffraction Imaging with MCMC
}

\author{
C. David Williams ${ }^{1}$, Magdalena Balazinska ${ }^{1}$, and Thomas L. Daniel ${ }^{1}$ \\ University of Washington, Seattle WA 98195, USA, \\ cdave@uw.edu
}

\begin{abstract}
High-speed X-ray diffraction is the state-of-the-art approach to understanding protein structure and dynamics in living tissues, especially muscles. Existing analytic approaches, however, require expert hand-digitization to extract parameters of interest. This produces repeatable measurements, but remains subjective and does not offer information on the precision of the measured parameters or strict reproducibility of analyzed data. We developed a processing tool chain, which first segments the diffraction image into regions of interest using highly conserved features and then samples the possible parameter values with a Markov chain Monte Carlo approach. Our approach produces an automated, reproducible, objective estimate of relevant image parameters.
\end{abstract}

Keywords: image analysis, X-ray diffraction, muscle structure, MCMC modeling

\section{Motivation}

The molecular regulation of force generating protein systems remains a fundamental open problem in biology. Muscle uses a highly organized lattice of interacting elastic and force generating molecules to create controlled macroscale movement $[9,8]$. The advent of advanced X-ray diffraction imaging lets us analyze protein motions at spatial and temporal scales never previously realized, giving novel insight into the molecular basis of motion in living systems.

Exploring the dynamics of protein interactions in muscle requires imaging that provides information at the nanometer to Angstrom scale. X-ray diffraction provides that information and is unique in its ability to image live muscle during movement, where other techniques (e.g. solution biochemistry or cryo electron microscopy) are only able to resolve properties of isolated individual molecular motors or anatomic features of static, dried muscle. This ability to image in vivo makes $\mathrm{X}$-ray diffraction the only direct means of measuring the structural changes that generate force; all other techniques that permit some level of molecular motor activity require the removal of those motors from the regulating structure of the thick/thin filament lattice and are thus less informative about the processes that actually control activation. This is a key advantage of X-ray diffraction: changes in muscle microstructure are highly correlated with changes 

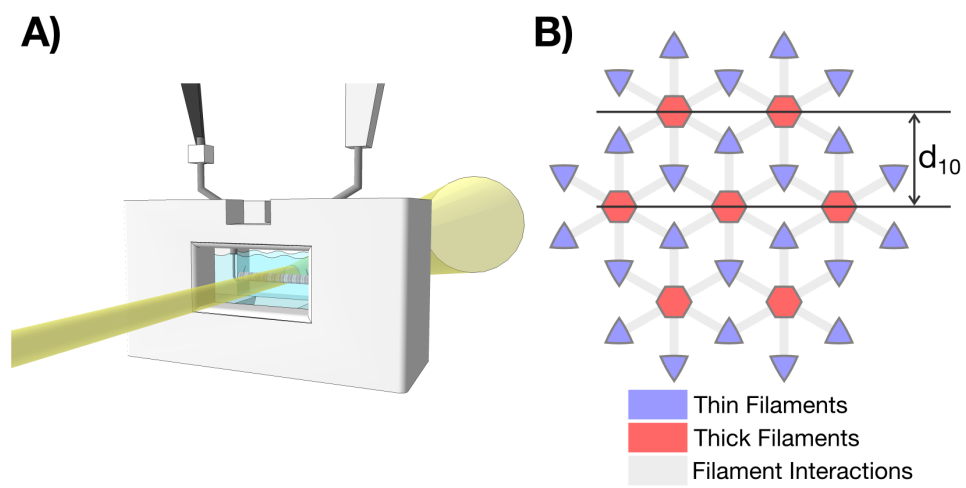

Fig. 1. Data collection and interpretation. A) Diffractive imaging of an isolated muscle sample requires a physiological solution bath with top entry for mounting to force/length controllers (arms) and front and rear cut outs to allow the X-ray beam to pass through and scatter off the muscle sample. B) Muscle's contractile lattice is composed of thick and thin filaments. When viewed down the long axis, these filaments form a hexagonal array. The $d_{10}$ spacing is the distance between adjacent lines of thick filaments, and is proportional to the distance molecular motors (gray links) must diffuse across in order to bind.

in force production and any attempt to separate the two removes significant aspects of the system's response to real-world stimuli $[3,1]$.

Surprisingly, we still use human experts to manually extract structural parameters from X-ray images with NIH ImageJ via manual selection of peaks and rock-of-eye parameter fitting. This produces repeatable measurements but is subjective, fails to provide confidence intervals for the measured values, and is not reproducible by naive digitizers. Additionally hand digitization is a timeintensive analytic technique, with a single digitizer able to process only a few hundreds of images a day. Historically this rate has been sufficient, but new highspeed imaging systems let us investigate short-timescale components of muscle contraction and generate data sets with many thousands of images. The need for an automated and reproducible image analysis tool chain is clear.

We seek to build a service for the automated analysis of muscle structure X-ray images. Users should specify the analysis they need using a declarative query interface and the system should automatically process the user's image database. In this work, we present the first components of the processing tool chain at the heart of this service. The toolchain first segments the diffraction image into regions of interest using conserved features and then samples the possible parameter values with a Markov chain Monte Carlo approach.

\section{Prototype}

We focus initially on measuring the $d_{10}$ parameter, a crucial spacing in muscle shown in Figures 1 and 2 . The $d_{10}$ spacing determines the distance which muscle's 

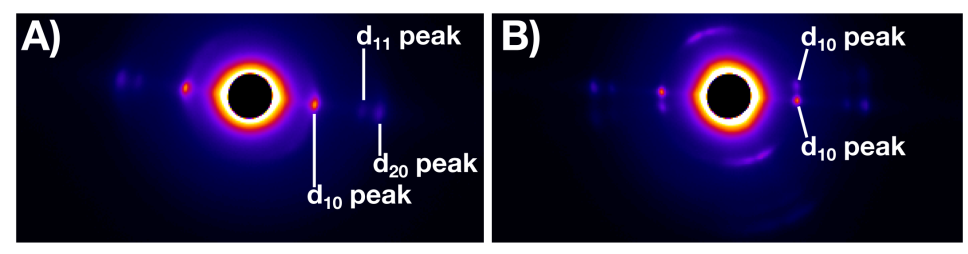

Fig. 2. X-ray diffraction images. A) An X-ray diffraction image exhibits $d_{10}, d_{11}$, and $d_{20}$ peaks; the first and last are proportional to the distance between adjacent rows of thick filaments. B) In this image, two fibers at slight angles to each other have generated multiple $d_{10}$ peaks which must be segregated during the detection process. Such two-fiber exposures occur rarely in the test corpus used in this work and are successfully processed by the automated grouping of peaks.

molecular motors must bridge in order to bind and generate force [10]. This distance changes during contraction and thus regulates the force produced [5].

Images generated during experiments share several key features, which serve as challenges or fiduciary marks during analysis. As seen in Figure 2, the brightest part of the image background is occluded by a circular stop. This physical block prevents damage to the detector from the high photon flux at the center of the X-ray beam. Surrounding the blocked region, the remainder of the image displays an exponentially decaying background. We must locate and model the symmetric pairs of diffraction peaks interrupting the exponential background. Our core data analysis pipeline includes two steps: image segmentation and image modeling with MCMC processes.

\subsection{Image segmentation}

The system first identifies the dark central circular blocked region to act as a relative landmark for subsequent operations. Consistent with experimental design, we assume it contains the center of the diffraction pattern. The edge of the background surrounding the block is the brightest region, so the system first splits the image between areas with values less than and greater than two standard deviations above the mean. This partitioning yields a binary image where the center blocked region is surrounded by a halo of the upper end of the pattern background and occasional dots where diffraction peaks rise more than two standard deviations above background. We convert this binary image to a hierarchical contour set with OpenCV, an open source library of standard image processing techniques [2]. We then take the blocked region to be the inner-most contour and model it as the smallest enclosing circle, shown as light green in Figure 3B.

With the central blocked region located, we identify local maxima in three by three groups after Gaussian smoothing with kernel having a three pixel standard deviation. We reject resulting maxima in regions unlikely to provide peaks of interest before attempting to match peak pairs. Masked rejection-regions consist of: 1) a circular zone around the central blocked region where the blocking 


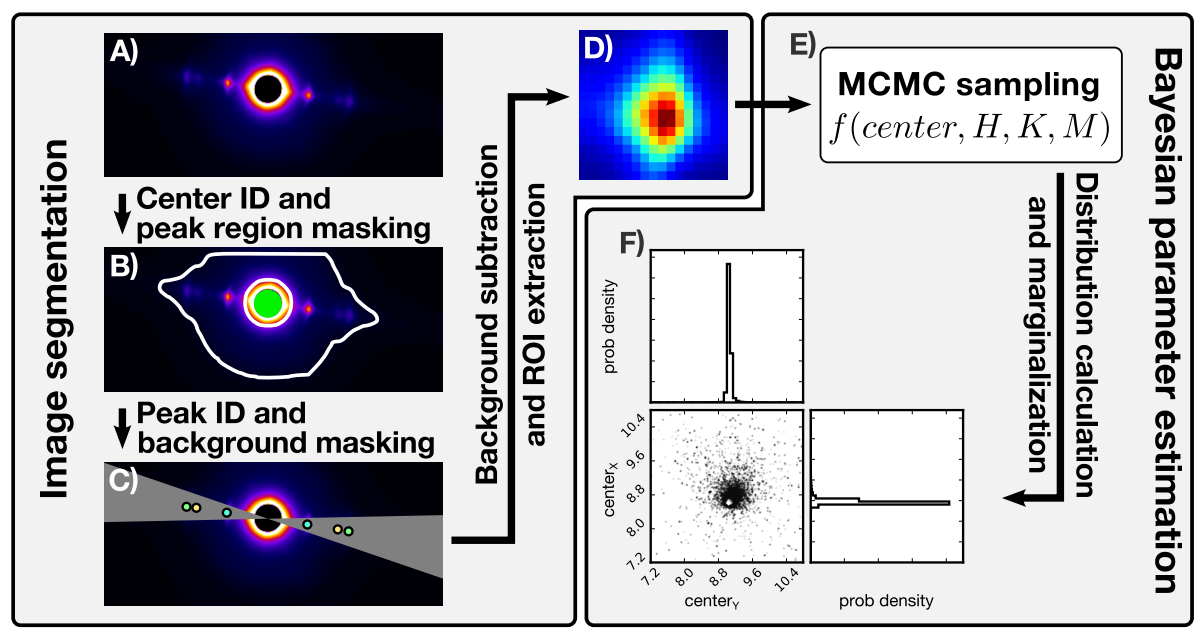

Fig. 3. Analysis workflow. A) An X-ray diffraction image is read in. B) The blocked center region (in green) is detected and used as a landmark. Low signal regions, the central blocked region, and image edges are masked off, leaving only the foreground outlined in white. C) The image is smoothed and peaks are detected, paired, and classified. To remove the exponentially decaying background from the peaks we mask off the area around the diffraction lines, shown in gray. The remaining background is then collapsed into a radial profile centered at the mean peak position. A double exponential fit to this profile is computed and subtracted from the regions of interest surrounding the $d_{10}$ peaks, leaving just the peak signal shown in D). E) We use an MCMC sampler to calculate the parameters generating the distributions these peaks are sampled from. F) We marginalize across peak parameters other than those of interest, extracting peak positions and the error in those position estimations.

generates non-peak local maxima, 2) areas below the 80th percentile where detector noise dominates, 3 ) areas near the image edge where peaks are partially cropped. The resulting unmasked area from which we keep maxima is bounded by a white border in Figure 3B.

Next, we cluster maxima into peak pairs based on their distance and angle from the center of the blocked region. Starting with those maxima nearest the blocked region, a corresponding maximum is sought an equal distance away from the blocked region and located so that the angle formed by the two maxima and the center of the blocked region is $180^{\circ}$. In cases where the matching maxima is clipped by the image frame or fails to be distinguished from background the initial maximum is discarded. For a maxima to be considered a match its distance and angle must match those of the initial maxima within $10 \%$. With peak pairs now identified (shown as color matched dots in Figure 3C), the diffraction center is identified by taking the mean location between peak pairs and the background is removed.

We subtract the background by first masking arcs encompassing peak pairs and then fitting a double exponential to a radial profile of the remaining image. 
An arc swept $12^{\circ}$ out on either side of each peak pair masks the effect of the diffraction peaks on the background (shown as a light gray arc under the peak pairs in Figure $3 \mathrm{C}$ ). We calculate a radial profile of the image around the pattern center, omitting diffraction line regions. We fit a double exponential function of the form background $=a+b e^{-x c}+d e^{-x e}$ to the radial profile. From these parameters we generate an estimated background image and subtract it from the real image, allowing us to extract the $d_{10}$ peaks as regions of interest (ROIs) unhindered by an overlaid diffraction background.

\subsection{Image modeling with MCMC processes}

With the background subtracted and the $d_{10}$ peaks identified and isolated from the rest of the image as ROIs, we apply Markov chain Monte Carlo (MCMC) sampling to determine the probability distributions from which the peak parameters could be drawn. We treat the peaks as being drawn from an underlying Pearson VII distribution, commonly used to fit X-ray diffraction peaks [7]. This process allows us to generate possible peak matches using five parameters: peak center x-location, peak center y-location, peak height, peak spread, and peak decay rate. We perform an initial peak fitting by residual minimization between a generated peak and the extracted ROI. This gives a set of starting parameters that we use, with random variation, to initialize the positions of the MCMC agents that will explore the model space.

Before MCMC sampling we must define our query's likelihood and prior. We choose a flat prior as our initial information about the model is minimal. To calculate the likelihood we represent each pixel's photon count as a Poisson process in the form $P(d \mid m)=e^{-m}\left(m^{d} / d\right.$ !) where $m$ is the model value and $d$ is the experimental data value. These functions are fed into emcee, an efficient MCMC analysis Python library [4]. After a burn in period of 100 steps, the sampler histories are erased and a further 1000 steps are run to generate the posterior probability distributions of our peak parameters.

One of MCMC modeling's convenient features is that extracting only a subset of parameters marginalizes across those we discard. That is, when we are interested in only the $\mathrm{x}$ - and $\mathrm{y}$-locations of the peak center to precisely calculate $d_{10}$ spacing (as in Figure $3 \mathrm{~F}$ ), we automatically integrate our uncertainty about peak height, spread, and decay.

\section{Preliminary Evaluation}

We apply our workflow to a test corpus of 1,220 images generated using X-ray diffraction during insect flight muscle research at the Argonne National Laboratory BioCAT Beamline. Sample high-quality and challenging images are shown in Figures 2A and 2B [6]. We tested the output of our automated system against the results from a domain expert manually digitizing peak locations. Our initial image segmentation step, described in Section 2.1, successfully identifies the center blocked region of each image in greater than $99 \%$ of images in our 

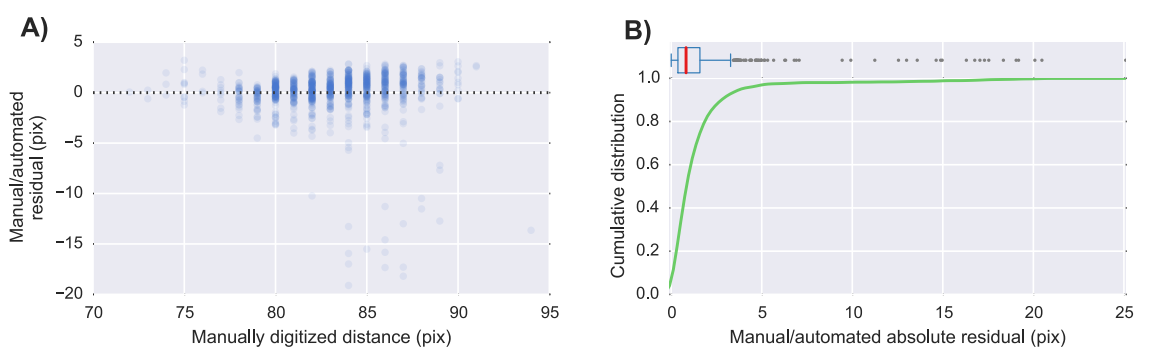

Fig. 4. Validation against hand-measurement. A) The majority of residuals (between the manually and automatically measured $d_{10}$ peak distances) are within a single pixel of the manually digitized validation values. In a handful of outliers the automated method mis-identifies a closer structure as the $d_{10}$ pair. B) The cumulative distribution of the residuals shows that half of the automated measurements agree within 0.8 pixels and over $90 \%$ agree within 3 pixels. A quartile box plot above shows the heavily leftweighted asymmetry of the residual distribution.

test corpus. The overall process allows us to calculate peak-to-peak distances to sub-pixel accuracy with a confidence interval of $90 \%$, with residuals between expectation values and manually digitized peak positions shown in Figure 4. MCMC sampling combined with image segmentation allows us to precisely, accurately, and automatically locate the $d_{10}$ peak centers and thus calculate the lattice spacing measured by a diffraction image to within $0.03 \mathrm{~nm}$.

Because the images in our corpus are a standard sample of those produced by high-speed X-ray diffraction, our positive preliminary results are a strong indication for the potential of this approach.

\section{Challenges and Next Steps}

Our initial data processing pipeline produces an automated, reproducible, objective estimate of relevant image parameters but the following challenges remain:

- Development of a declarative language to describe processing steps will speed use of this technique and ease reproducibility. The key question is to define the types of operations that users should be able to specify and how to specify them. Our goal is to generalize to a broad set of analysis needs for $\mathrm{X}$-ray images of muscle structure.

- Packaging of this tool chain into a cloud deployable containerized image will enable trivial scaling to work with larger datasets. Furthermore, the ability to access to tool chain directly through a web browser with automatic backend deployment of the analysis pipeline will facilitate adoption.

- This toolchain works best when applied to sharp and well defined images produced by isolated fibers. When working with images produced by in vivo samples, such as those taken through a fly thorax, diffraction from multiple muscles in different orientations is common. These multiple-muscle images 
produce a more extreme version of the image shown in Figure 2B, where twin rotated peaks that are twisted and blurred about the center of the diffraction pattern and may have substantially divergent $d_{10}$ distances. Twin rotated peaks must be classified by diffraction line/muscle and treated independently during analysis.

- Application of these techniques to coming ultra-high temporal-resolution images with far lower signal:noise will strain autosegmentation and peakfitting techniques.

The increasing prevalence of advanced diffractive imaging techniques demands data analytic methods that can handle, objectively and reproducible, significant increases in the volume of data generated and analyzed. This toolchain is a first step in a process that will greatly increase the power and capabilities of a widely used approach to understanding protein structure in living tissue.

\section{Acknowledgments}

This work was supported in part by NSF grant IIS-1110370, the Intel Science and Technology Center for Big Data, the Army Research Office through ARO Grants W911NF-13-1-0435 and W911NF-14-1-0396, an award from the Gordon and Betty Moore Foundation and the Alfred P Sloan Foundation, the Washington Research Foundation Fund for Innovation in Data-Intensive Discovery, and the UW eScience Institute.

We thank Jake VanderPlas for helpful discussions of statistical techniques,

Tom Irving for advice on X-ray imaging, Gideon Dunster for digitization of diffraction images, and Simon Sponberg for the sharing of diffraction images.

\section{References}

1. M. A. Bagni, G. Cecchi, P. J. Griffiths, Y. Maeda, G. Rapp, and C. C. Ashley. Lattice spacing changes accompanying isometric tension development in intact single muscle fibers. Biophys J, 67(5):1965-75, Nov 1994.

2. G. Bradski. OpenCV: an open source computer vision library. Dr. Dobb's Journal of Software Tools, 2000.

3. B. Brenner and L. C. Yu. Equatorial x-ray diffraction from single skinned rabbit psoas fibers at various degrees of activation. changes in intensities and lattice spacing. Biophys J, 48(5):829-34, Nov 1985.

4. D. Foreman-Mackey, D. W. Hogg, D. Lang, and J. Goodman. emcee: The mcmc hammer. PASP, 125(925):306-12, Jan 2013.

5. N. T. George, T. C. Irving, C. D. Williams, and T. L. Daniel. The cross-bridge spring: can cool muscles store elastic energy? Science, 340(6137):1217-20, Jun 2013.

6. T. C. Irving. X-ray diffraction of indirect flight muscle from drosophila in vivo. Nature's Versatile Engine: Insect Flight Muscle Inside and Out. JO Vigoreaux, editor. Landes Bioscience, Georgetown, TX, pages 197-213, 2006. 
7. M. M. H. Jnr, V. G. Veeraraghavan, H. Rubin, and P. G. Winchell. The approximation of symmetric x-ray peaks by pearson type vii distributions. J. Appl. Cryst., 10:66-68, Jan 1977.

8. B. M. Millman. The filament lattice of striated muscle. Physiol Rev, 78(2):359-91, Apr 1998.

9. M. K. Reedy, K. C. Holmes, and R. T. Tregear. Induced changes in orientation of the cross-bridges of glycerinated insect flight muscle. Nature, 207(5003):1276-80, Sep 1965.

10. C. D. Williams, M. K. Salcedo, T. C. Irving, M. Regnier, and T. L. Daniel. The length-tension curve in muscle depends on lattice spacing. Proc Biol Sci, 280(1766):20130697, Dec 2013. 